\title{
The Motion of Slender Particles in Shear Flow
}

\author{
Woo Il Lee and George S. Springer
}

\begin{abstract}
Department of Mechanical Engineering \& Applied Mechanics
The University of Michigan, Ann Arbor, Michigan 48109
\end{abstract}

(Received July 12, 1982)

\begin{abstract}
A model was developed for calculating the motion of dilute suspensions of rigid, slender particles in a uniformly sheared viscous liquid, taking into account interactions between particles. The model was applied to the plane Couette flow problem in which particles are suspended in a liquid moving parallel to a plane with a uniform, shearing motion. The results of the model were compared to existing data, and good agreement was found between the calculated and experimental results. Using the model, results were also obtained which illustrate the major effects of particle-particle interactions on the angular motion of the particles.
\end{abstract}

\section{INTRODUCTION}

Tumerous investigators have addressed the problem of the motion of par1 ticles suspended in a flowing liquid (e.g. see the summaries [1-3]). However, the existing analytical results pertain only to single particles. Analytical results are as yet unavailable for flows in which particles interact with each other. The objective of this investigation was, therefore, to develop a model which describes the motion of slender particles suspended in a sheared, viscous fluid, taking into account particle-particle interactions.

The basic concepts of the model are described first. The model is then applied to the problem of dilute suspensions of slender particles suspended in a

J. REINFORCED PLASTICS AND COMPOSITES, Vol. 1 (October 1982), p. 279 
fluid moving parallel to a plane with a uniform shearing motion (Couette flow). This problem was selected for detailed analysis because data exist which can be used for comparison with results of the model.

\section{THE PROBLEM}

The following problem was investigated. Particles are suspended randomly in a Newtonian liquid. The flow field of interest is large in comparison with the particle size and interactions between particles and the walls bounding the fluid are neglected. The viscosity of the liquid is high, so that the inertia forces acting on the particles are negligible compared to the viscous forces. The particles have the same density as the liquid. The concentration of the particles is low, so that a) the mean velocity field of the liquid is not influenced by the presence of the particles, b) the viscosity of the particle-liquid mixture is the same as the liquid viscosity, and c) only two particles may interact at any given time. The particles are rigid rods, their shapes being approximated by prolate spheroids. The aspect ratio of each particle is

$$
r_{e}=\frac{a}{b}
$$

where $a$ and $b$ are the lengths of the major and minor axes, respectively. The particles are not necessarily of the same size. The aspect ratio may be different for different particles, but for each particle the condition $\mathrm{r}_{\mathrm{e}} \gg 1$ must be met.

At time $\mathrm{t}=0$ a known velocity gradient is imposed on the liquid. This velocity gradient is uniform and constant throughout flow field of interest

$$
G_{i j} \equiv \frac{\partial u_{i}}{\partial x_{j}}=\text { const. }
$$

where $i$ and $j$ represent coordinate directions (Fig. 1). The components of the mean flow velocity $u_{i}$ vary with position in the following manner.

$$
u_{i}=\sum_{j=1}^{3} \frac{\partial u_{i}}{\partial x_{j}} x_{j}
$$

The shear stress resulting from the flow causes the particles to undergo translational and rotational movements. The problem is to determine the positions of each particle and the average orientation of all particles as functions of time. The average orientation may be expressed by the mean projections of the particles on the $x_{1}, x_{2}, x_{3}$ axes (Fig. 1)

$$
\bar{X}_{1}=\frac{1}{N} \sum_{j=1}^{N} \sin \theta_{j} \cos \phi_{j}
$$




$$
\begin{aligned}
& \bar{X}_{2}=\frac{1}{N} \sum_{j=1}^{N} \sin \theta_{j} \sin \phi_{j} \\
& \bar{X}_{3}=\frac{1}{N} \sum_{j=1}^{N} \cos \theta_{j}
\end{aligned}
$$

The translational velocity of each particle is assumed to be the same as the fluid velocity at the location coinciding with the center of mass of the particle. Particle-particle interactions are assumed to alter only the angular motion of the particle but not its translational motion. Hence, by knowing the fluid velocity, the translational motion and the position of each particle can readily be calculated. Thus, the problem is to determine the average angular motion of the particles, expressed in terms of the mean axial projections (eqs. 4).

\section{THE MODEL}

In the model proposed here the motion of each particle is followed. At any given instant a particle must either be in "collision" with one other particle or is moving without interference from any other particle. The collision condition is defined subsequently. The angular motion (angular velocity $\underset{\omega}{\omega}$ and angular position $\phi$ and $\theta$, Fig. 1) of each particle is then calculated according to one of two procedures.

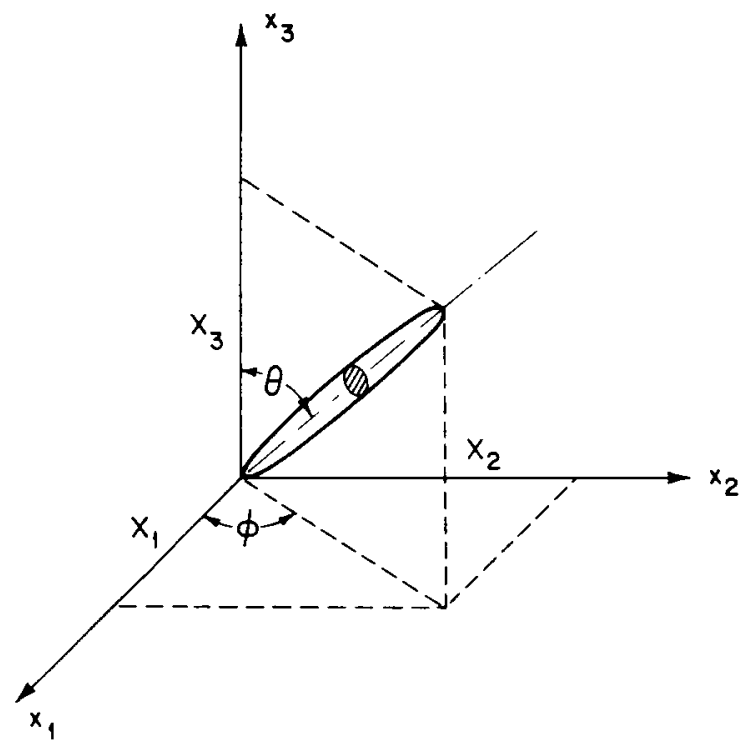

Figure 1. Coordinate system used in the calculations. 
(i) In the absence of "collision" the instantaneous angular motion of each particle is calculated using Jeffery's results [4] summarized in the appendix.

(ii) When two particles "collide" the angular velocity and orientation of each particle after the collision as e calculated on the basis of two assumptions regarding the velocities at the points of contact (A or B, Fig. 2). The contact point is defined as the intersection of the line coinciding with the major axis (line $1_{A}$ or $1_{B}$ ) and the line perpendicular to both $1_{A}$ and $1_{B}$ (line $L)$. The "contact line" $L$ is the shortest line between $1_{A}$ and $1_{B}$.

The velocity components normal to the contact line remain unchanged during the collision

$$
\underset{\sim}{V_{n A}^{\prime}}=\underset{\sim}{V_{n A}} \text { and } \underset{\sim}{V_{n B}^{\prime}}=\underset{\sim}{V_{n B}}
$$

The prime indicates velocities after the collision.

After collision the velocity components parallel to the contact line are the same for both particles and have the value equal to the average of the two normal velocity components just prior to the collision

$$
\underset{\sim P A}{V_{P A}^{\prime}}=\underset{\sim P B}{V_{P B}^{\prime}}=\underset{\sim}{V_{P}^{\prime}}=\frac{1}{2}\left(\underset{\sim P A}{V_{P A}}+\underset{\sim P B}{V}\right)
$$

Expressions suitable for calculating the positions of the contact points, the angular velocities, and the angular positions of the particles are given in the appendix.

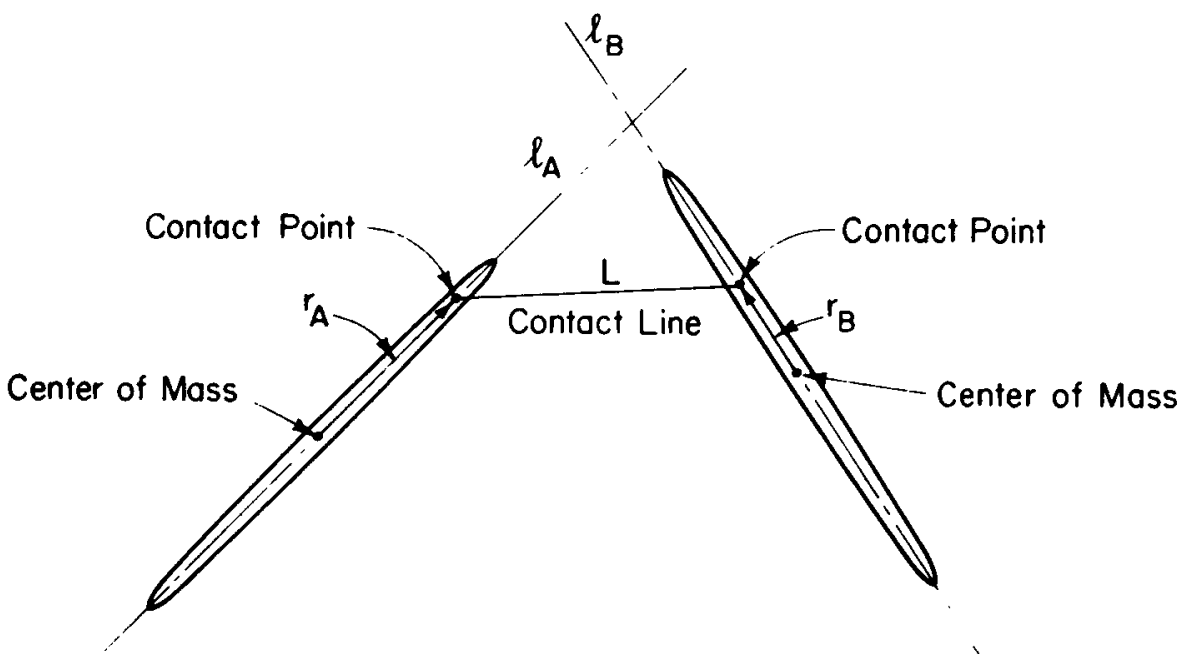

Figure 2. Geometry during collision of particle $A$ and particle $B$. 
In order to establish whether or not two particles are colliding, a "probability of collision" $P_{c}$ is defined

$$
P_{c}=\frac{\alpha^{m}}{(s / a+\alpha)^{m}}
$$

where $\alpha$ and $m$ are constants and $s$ is the distance between the centers of masses of the two particles under consideration. Two particles are taken to be colliding (and their angular motions are calculated according to step ii above) when the probability $\mathbf{P}_{\mathrm{c}}$ is greater than some random number $\mathbf{R}$

$$
P_{c}>R \rightarrow \text { collision }
$$

Two particles are taken to be moving independently (and their angular motions are calculated by Jeffery's results, step i) when the probability $P_{c}$ is less than some random number $\mathbf{R}$

$$
P_{c}<R \rightarrow \text { no collision }
$$

The constants $\alpha$ and $m$ may depend on the aspect ratio. The values of these constants must be determined by matching the results of the model to data. An estimate of the value of $\mathrm{m}$ can be made by observing that-according to Jeffery's results - the disturbance in the mean velocity field due to the presence of a particle $|\Delta y| /|u|$ decays inversely with the cube of the distance from the particle. With the value $m=3$ the probability of collision $P_{c}$ also decreases in inverse proportion to the cube of the distance. Accordingly, eq (7) becomes

$$
P_{c}=\frac{\alpha^{3}}{(s / a+\alpha)^{3}}
$$

\section{METHOD OF SOLUTION}

The model described above, together with the expressions given in the appendix, can be used to calculate the motion of the particles. Solutions must be obtained by numerical methods. To facilitate the calculations the liquid region of interest is enclosed by a control volume (Fig. 3). Number of particles are inserted into the control volume in a random manner. The size of each particle must be specified. The dimensions of every particle may be the same or may be different. In practical situations the aspect ratios of the particles frequently vary about a mean $\bar{r}_{e}$ in a Gaussian manner. In this case the probability distribution of $r_{e}$ may be expressed as [5].

$$
g\left(r_{e}\right)=\frac{1}{\sqrt{2 \pi \sigma}} \exp \left[-\frac{\left(r_{e}-\bar{r}_{e}\right)^{2}}{2 \sigma^{2}}\right]
$$

By specifying the standard deviation $\sigma$, the mean value $\bar{\Gamma}_{e}$, and the total number of particles N, Box-Muller's transformation [6] can be used to obtain $r_{e}$ values 


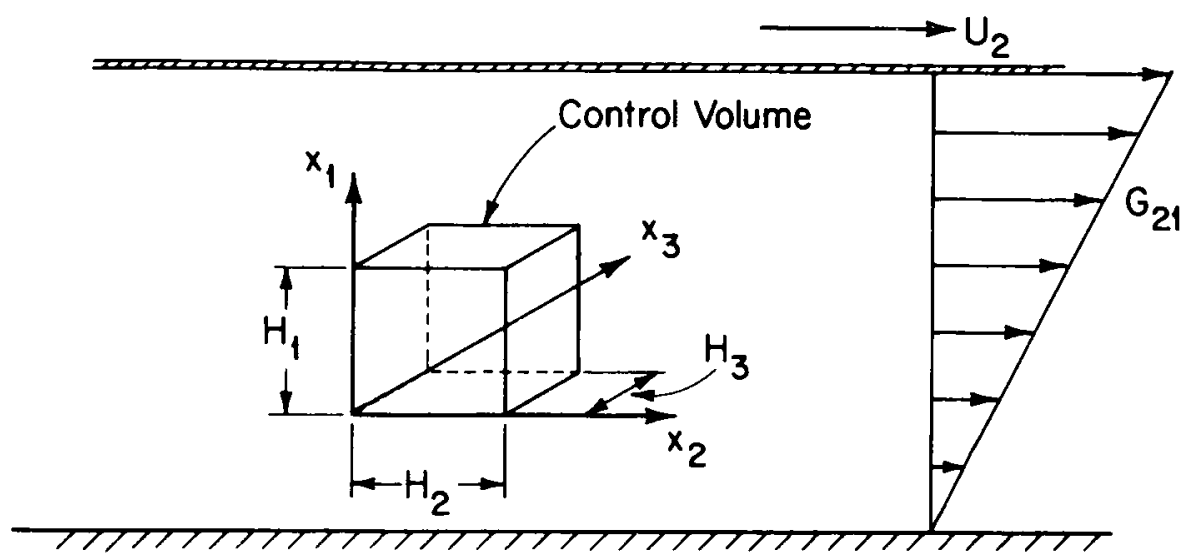

Figure 3. Plane Couette flow problem and control volume used in the calculations.

for each particle so that the above distribution (eq 11) is satisfied. Preprogrammed computer subroutines are available for such calculations.

The initial position of the center of mass $\mathrm{xcm}$ and the initial orientation $(\phi, \theta)$ of each particle may be selected with the aid of random numbers $\left(R_{\mathbf{i}}-R_{s}\right)$

$$
\begin{aligned}
x_{1}^{\mathrm{cm}} & =H_{1} R_{1}, x_{2}{ }^{\mathrm{cm}}=H_{2} R_{2}, x_{3}{ }^{\mathrm{cm}}=H_{3} R_{3} \\
\phi & =2 \pi R_{4}, \theta=\cos ^{-1}\left(1-2 R_{5}\right)
\end{aligned}
$$

where $H_{1}, H_{2}$, and $H_{3}$ are the dimensions of the control volume. The velocities, positions, and orientations of every particle are then calculated at subsequent small time intervals using eqs (A.7)-(A.14) for particles moving freely, and eqs (A.2)-(A.6) for particles undergoing collisions.

For a particle moving freely the motion of the particle depends on the initial $(t \leqslant 0)$ orientation of the particle. This initial orientation is specified by the "orbit constant" $C$. The value of $\mathrm{C}$ is calculated for each particle [7].

$$
C=\frac{\tan \theta\left(r_{e}^{2} \cos ^{2} \phi+\sin ^{2} \phi\right)^{1 / 2}}{r_{e}}
$$

$\mathrm{C}$ is then taken to remain constant until a collision occurs. After collision a new value of $C$ is calculated for each of the two colliding particles.

The collision condition for each particle is determined by calculating the collision probability $P_{c}$ (eq 10$)$ between the particle under consideration and the particle nearest to it, and by comparing this probability to a random number (eqs 8 and 9). Because of the assumption of a dilute suspension collision of a particle with any particle other than its nearest partner is neglected.

The mean axial projections are calculated at each time step using eq (4).

Because of their translational motion particles may move out of the control 
volume. To maintain a constant particle concentration (i.e. a constant number of particles in the control volume) a new particle is introduced for each leaving particle. The $x_{1}$ and $x_{2}$ coordinates of each new particle is again selected randomly with the aid of random numbers. The $x_{2}$ coordinates are selected to correspond to the distance travelled by the leaving particle beyond the control volume.

$$
x_{1}^{\mathrm{cm}}=H_{1} \sqrt{R}, x_{2}^{\mathrm{cm}}=\left(x_{2}^{\mathrm{cm}}\right)_{L}-I\left(\frac{\left(x_{2}^{\mathrm{cm}}\right)_{L}}{H_{2}}\right) H_{2} \text { and } x_{3}^{\mathrm{cm}}=H_{3} R
$$

where $I$ indicates the integer part of the term in paranthesis and $\left(x_{2} \mathrm{~cm}\right)_{L}$ is the coordinate of the leaving particle. For convenience, the orientation of the new particle is assumed to be the same as that of the particle which left the control volume.

\section{RESULTS}

Using the model described previously, mean axial projections were calculated for particles suspended in plane Couette flow. The mean flow velocity, $u_{2}$ was parallel to the $x_{2}$ axis and the velocity gradient $\left(G_{21} \equiv G\right)$ was in the $x_{1} x_{2}$ plane (Fig. 3). A cube shaped control volume was used in the calculations. Sample calculations were performed with different numbers of particles in the control volume. It was found that the results did not change significantly when 100 or more particles were used. Therefore, all subsequent calculations were performed with 100 particles. The size of the control volume was then chosen to yield the required particle concentration.

Two sets of calculations were performed. First, results were generated for comparison with data. Second, results were obtained which illustrate the main effects of particle-particle interactions on the average orientation of the particles.

At a given time $t$, the average particle orientation depends on the velocity gradient $G$, the particle concentration $n$, the particle length $a$, and the aspect ratio $r_{e}$. The aspect ratio may be specified either by giving $r_{e}$ for each particle or, for Gaussian size distribution (eq 11), by specifying the mean $\bar{r}_{e}$ and the standard deviation $\boldsymbol{o}$.

In the first series of calculations results were obtained with. $G=0.5 \mathrm{~s}^{-1}$, $\mathrm{n}=20,50$ and $100 \mathrm{~cm}^{-3}, \mathrm{a}=0.72 \mathrm{~mm}, \overline{\mathrm{r}}_{\mathrm{e}}=11.6$, and $\sigma=3.6$ percent. These values correspond to the conditions which existed in Okagawa and Mason's experiments [8].

The mean projections on the $x_{1}$ axis as functions of time are shown in Fig. 4. In this, and in all subsequent figures, time is expressed in dimensionless form $t / T$, where $T$ is the period of rotation of a single particle [4].

$$
T=\frac{2 \pi}{G}\left(r_{e}+\frac{1}{r_{e}}\right)
$$



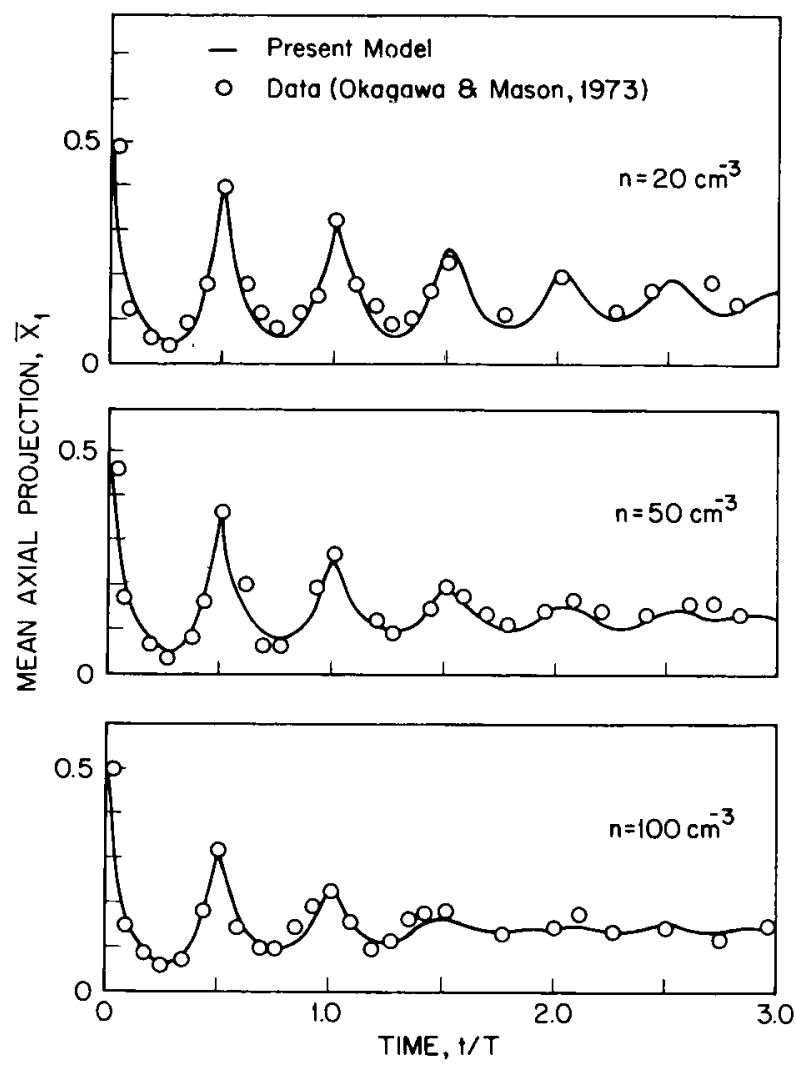

Figure 4. Comparisons between calculated and measured mean axial projections for different particle concentrations. $\left(G=0.5 \mathrm{~s}^{-1}, \bar{r}_{e}=11.6, \sigma=3.6 \%, a=0.72 \mathrm{~mm}, a=0.5\right.$ )

In Fig. 4 the data of Okagawa and Mason are compared to the results of the model computed with $a=0.5$. The excellent agreement between the results of the model and the data creates confidence in the validity of the model.

Anczurowski, Cox \& Mason [9] also obtained data for particles with a mean aspect ratio of 14.2. Anczurowski and his coworkers reported mean axial projections at four different times. These four data points are shown in Fig. 5. Results of the model (obtained again with $\alpha=0.5$ ) are also included in this figure. At $t / T=3$ there is good agreement between the result of the model and the data. This agreement further supports the model, and suggests that the constant $\alpha$ is insensitive to the value of $\bar{r}_{e}$, at least in the $\bar{r}_{e}$ range of about 10 to 15.

According to the model, the mean axial projections should remain constant after their steady state values are reached. On the other hand, the values of $\bar{X}_{1}$, $\overline{\mathbf{X}}_{2}$, and $\overline{\mathbf{X}}_{3}$, measured by Anczurowski et al changed after the oscillations were damped out (Fig. 5). The precise reason for these changes is unknown. The 


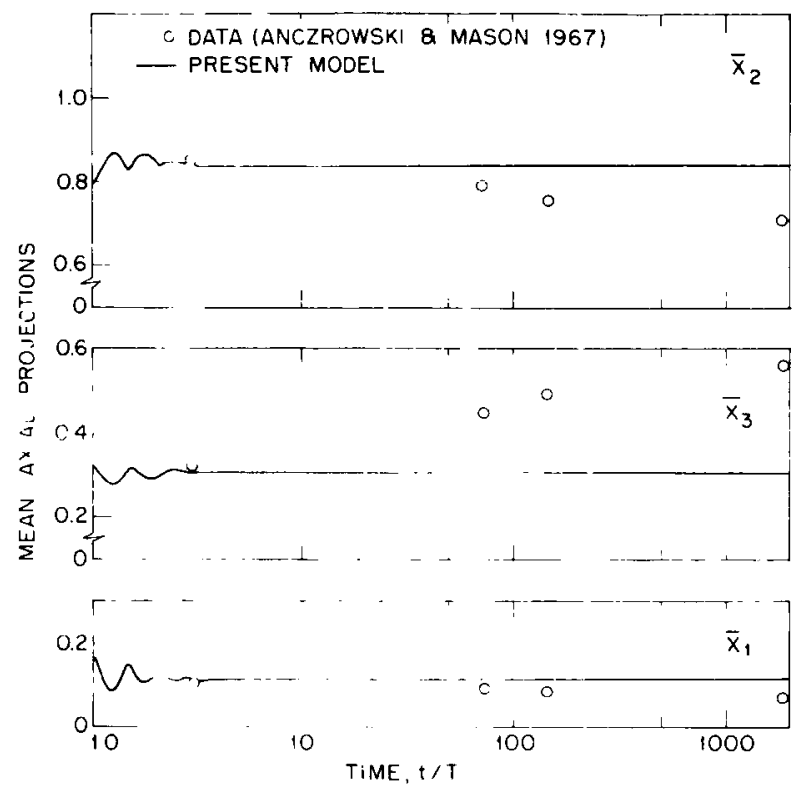

Figure 5. Comparison between calculated and measured mean axial projections. $\left(G=0.5 \mathrm{~s}^{-1}\right.$, $\left.\bar{r}_{e}=14.2,0=8.5 \%, a=0.362 \mathrm{~mm}, n=500 \mathrm{~cm}^{-3}, a=0.5\right)$

gradual changes in the mean axial projections may have been caused by a) nonNewtonian effects [10] or b) "long range" disturbances in the primary flow due to the particles.

Okagawa \& Mason [8] also reported data for particles with a mean aspect ratio of 3.96. In these experiments, initially $(t=0)$ the major axes of every particle were supposed to be parallel to the $x_{1}$ axes. Since the imposed velocity gradient $G$ was in the $x_{1} x_{2}$ plane, there should have been no collisions between the particles and the major axes of the particles should have remained in the $\mathrm{x}_{1} \mathrm{x}_{2}$ plane. However, in the experiments, particles collided and their axes did not remain in the $x_{1} x_{2}$ plane. The reason for this may have been that either the particles were not placed exactly in the plane of shear, or the particles were not slender or perfectly symmetric. Thus, unfortunately, these data are not suitable for comparison with the model.

The model is now used to illustrate the effects of collisions on the angular motion of the particles. A single particle suspended in unform flow rotates continuously in a periodic manner. Such a periodic motion is predicted by Jeffery's theory (eqs A.3-A.6), and has been demonstrated by experiments [7, 11-14]. The period of one complete rotation $T$ is given by eq (14). The angular velocity of the particle is not constant, but changes as the angle $\phi$ increases. Because of this non-uniform angular velocity, the mean axial projections of a large number of non-interacting particles (all particles having the same dimen- 

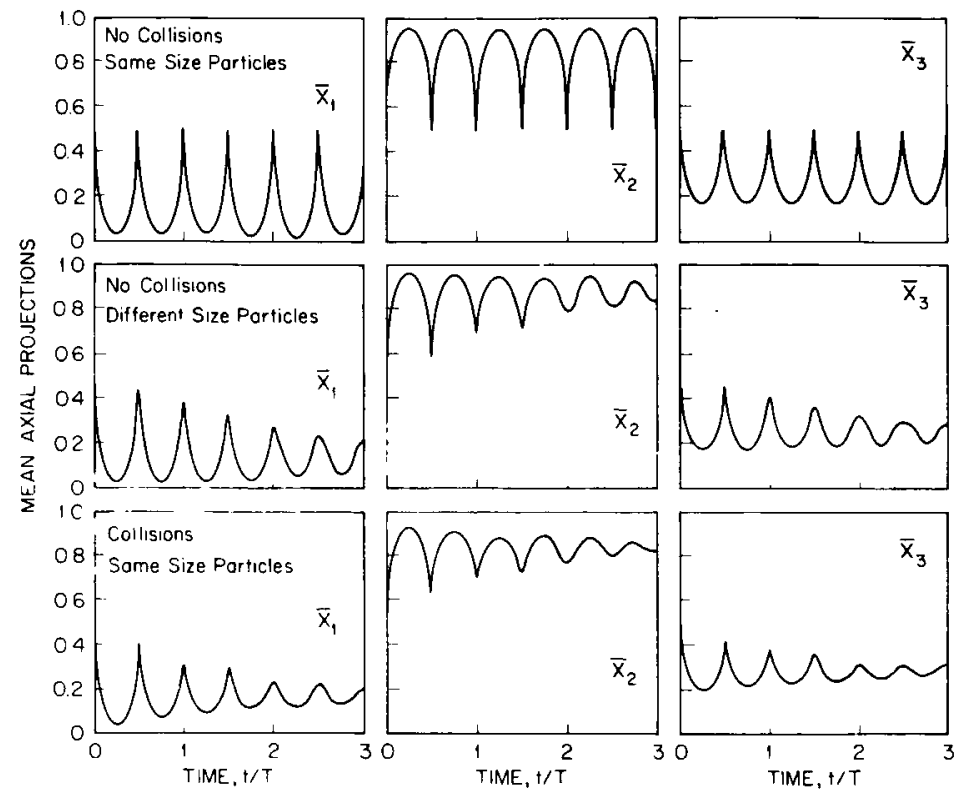

Figure 6. Mean axial projections as functions of time. Top: Large number of uniform size $(\sigma=0 \%)$ non-interacting particles. Initial particle orientation is random. Middle: Noninteracting particles having different sizes. $(0=3.6 \%)$ Bottom: Interacting particles all having the same dimensions. $\left(n=50 \mathrm{~cm}^{-3}, \alpha=0.5\right)$ For all cases $G=0.5 \mathrm{~s}^{-1}, \bar{r}_{\mathrm{e}}=11.6, a=0.72 \mathrm{~mm}$.

sion) also vary periodically, as illustrated in Fig. 6 . The period of oscillation is the same as the period of oscillation of a single particle. The amplitude of oscillations is approximately half the particle length and is constant. Since the mean axial projections are based on unit particle length (eq 4), the amplitude of oscillation of $\bar{X}_{1}$ is approximately $1 / 2$. The exact value of the amplitude depends on the aspect ratio.

The oscillations in $\overline{\mathbf{X}}_{1}$ become damped when particles interact with each other and when the aspect ratios of the particles are different. Such damping has been demonstrated experimentally [8] and is also predicted by the model, as illustrated by the typical results in Fig. 6. The oscillations die out (and the mean axial projections reach a steady state value) after a few rotations, in contrast to the undamped osciallations of non-interacting, uniform size particles.

It is interesting to note that the period of oscillation is practically unaffected by collisions or by differences in particle size (Fig. 6). On the other hand the rate of decay of the oscillations depends on both of these factors. The oscillations decay faster as the concentration (and consequently the number of collisions) increases (Fig. 7), as the particles become longer (Fig. 8), and as the differences in the aspect ratios of the particles (represented by the standard deviation) become larger (Fig. 9). The results in Fig. 10 further illustrate the effects 


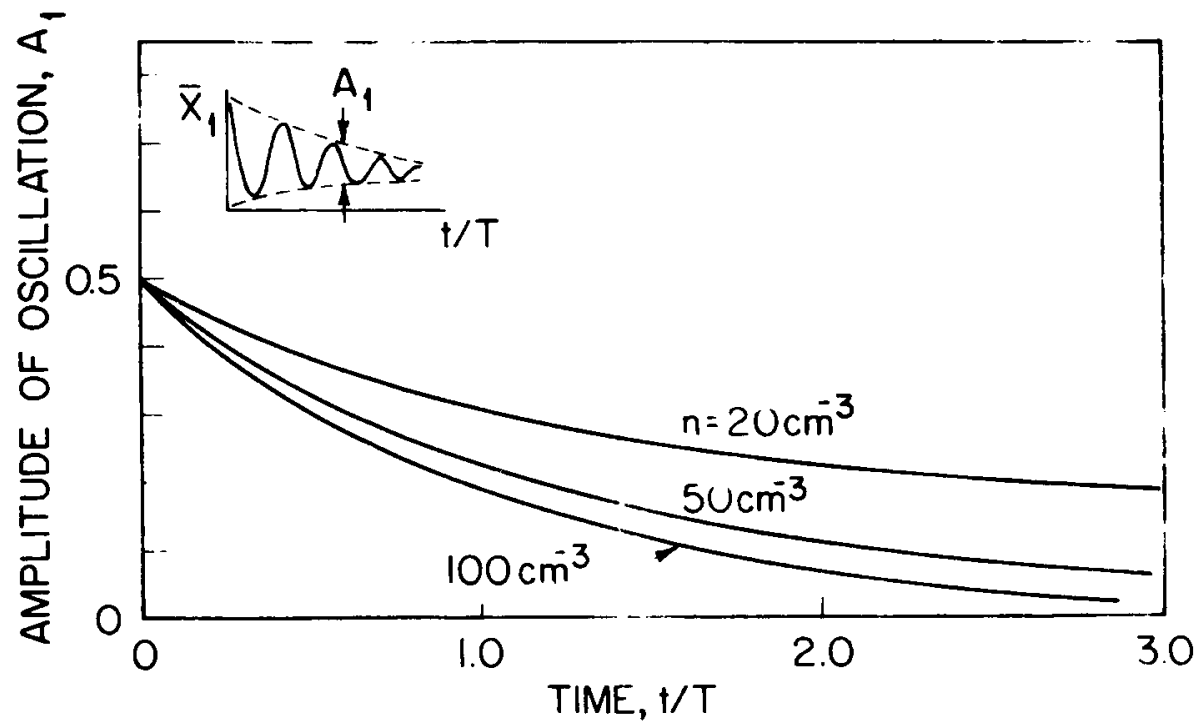

Figure 7. The effect of particle concentration on the amplitude of oscillation in the presence of particle-particle interactions. All particles have the same dimensions. $\left(\bar{r}_{e}=11.6, G=0.55^{1}\right.$, $a=0.72 \mathrm{~mm}, \alpha=0.5$ )

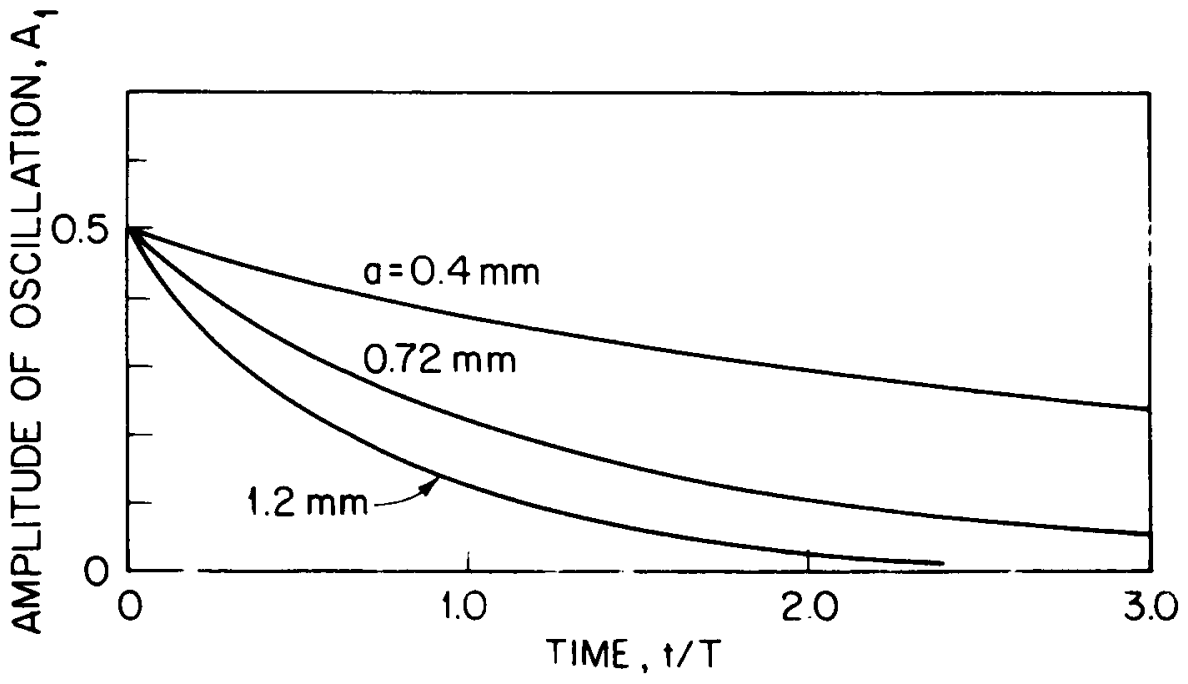

Figure 8. The effect of particle length on the amplitude of oscillation in the presence of particleparticle interaction. Every particle has the same ratio $\bar{r}_{e}=11.6 .\left(G=0.5 \mathrm{~s}^{-1}, n=50 \mathrm{~cm}^{-3}\right.$, $a=0.5) A_{1}$ is defined in Fig. 7. 
Woo Il Lee and George S. Springer

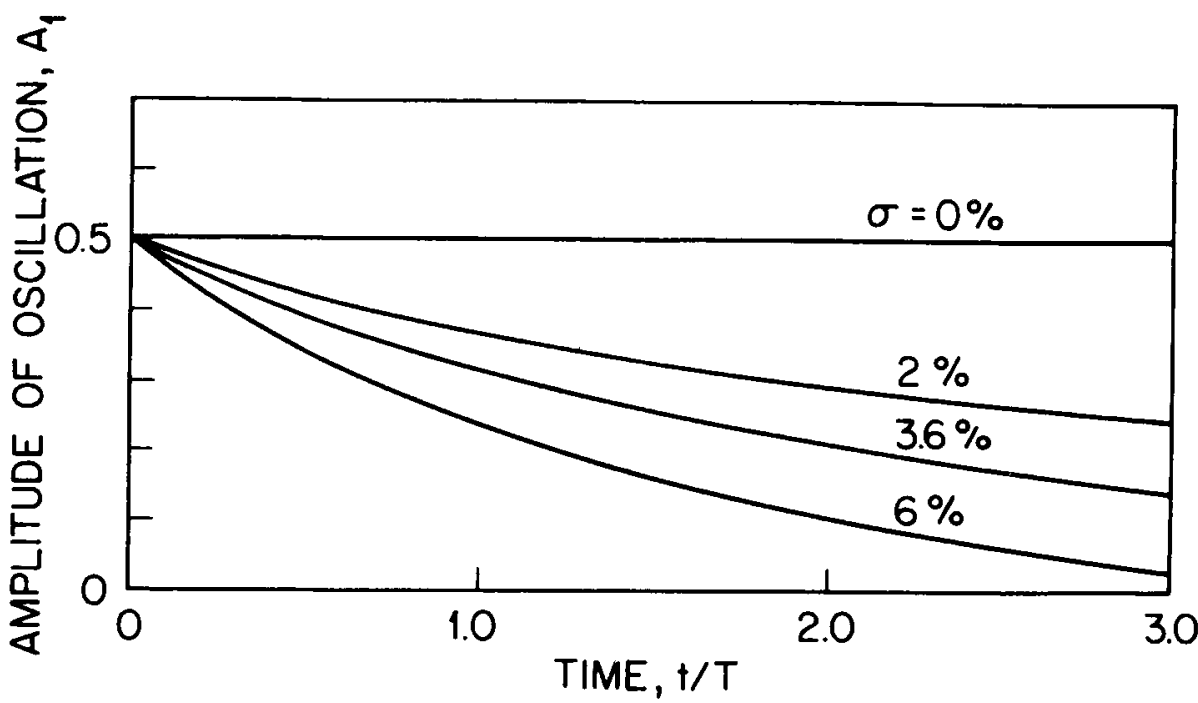

Figure 9. Changes in the amplitude of oscillation for a collection of non-interacting particles having different aspect ratio about the mean $\bar{r}_{e}$ (eq. II). $\left(\bar{r}_{e}=11.6, G=0.5 \mathrm{~s}^{-1}\right)$

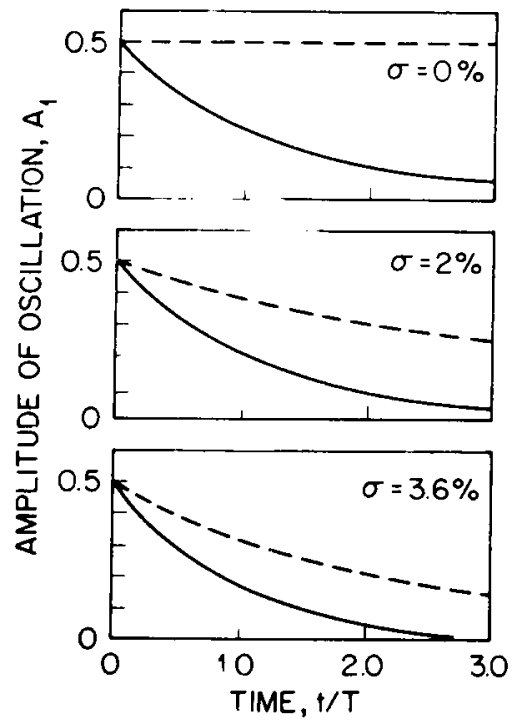

Figure 10. The amplitude of oscillation with and without taking into account particle-particle interactions.

Particle-particle interactions included in the calculations.

$\left.\overline{r_{e}}=11.6, G=0.5 \mathrm{~s}^{-1}, a=0.72 \mathrm{~mm}, n=50 \mathrm{~cm}^{-3}, a=0.5\right)$ 
of both collisions and $o$ on the rate of change in the amplitude of oscillations. The amplitude decays much faster when collisions occur than in the absence of particle-particle interactions. In Fig. 7-10 only the $\overline{\mathbf{X}}_{1}$ amplitude decays are shown. The amplitudes of $\bar{X}_{2}$ and $\bar{X}_{3}$ decay in similar manner.

The steady state values of the mean axial projections of interacting particles

$$
\bar{X}_{i}^{s s}=\frac{1}{N} \sum_{k=1}^{N}\left(X_{i}^{s s}\right)_{k}
$$

are related to the time averaged orientation of a single particle.

$$
<X_{i}>=\frac{1}{T} \int_{0}^{T} X_{i}(t) d t
$$

The relationship berween these two parameters could best be demonstrated by calculating $\bar{X}_{i}^{\text {ss }}$ and $\left\langle X_{i}>\right.$ and by comparing their values. Unfortunately, $\bar{X}_{i}$ depends on the initial orientation (i.e. on the orbit constant, eq 13) of the particle. Thus $\left\langle\mathbf{X}_{\mathbf{i}}\right\rangle$ does not have a unique value, and a direct comparison cannot be made between $\bar{X}_{i}{ }^{35}$ and $\left\langle X_{i}\right\rangle$. However, an indirect comparison can be made by comparing the number averaged steady state value of the angle $\bar{\phi}^{\text {ss }}$ for all those particles which have $\bar{\phi}^{s}$ values between 0 and $\pi / 2$

$$
\bar{\phi}^{s s}=\frac{1}{N} \sum_{i=1}^{N} \phi_{i}^{s s}
$$

to the time averaged value of $\langle\phi\rangle$ of a single particle

$$
<\phi>=\frac{1}{T / 4} \int_{0}^{T / 4} \phi d t
$$

where, for a single particle, $\phi$ is [4]

$$
\phi=\arctan \left[r_{e} \tan \left(\frac{2 \pi t}{T}\right)\right]
$$

Equations (19) and (20) show that $\langle\phi\rangle$ depends only on $r_{e}$. The nature of this dependence is shown in Fig. 11 where the values of $\langle\phi\rangle$, calculated by eqs (19-20), are given. The number of averaged steady state values of the angle (calculated by eq 18) are also included in this figure. As can be seen, for dilute suspensions such as considered here, $\langle\phi\rangle$ and $\bar{\phi}$ agree closely, impiying that the steady state values of the number averaged orientations of interacting particles are related to the time averaged orientation of a single particle. 


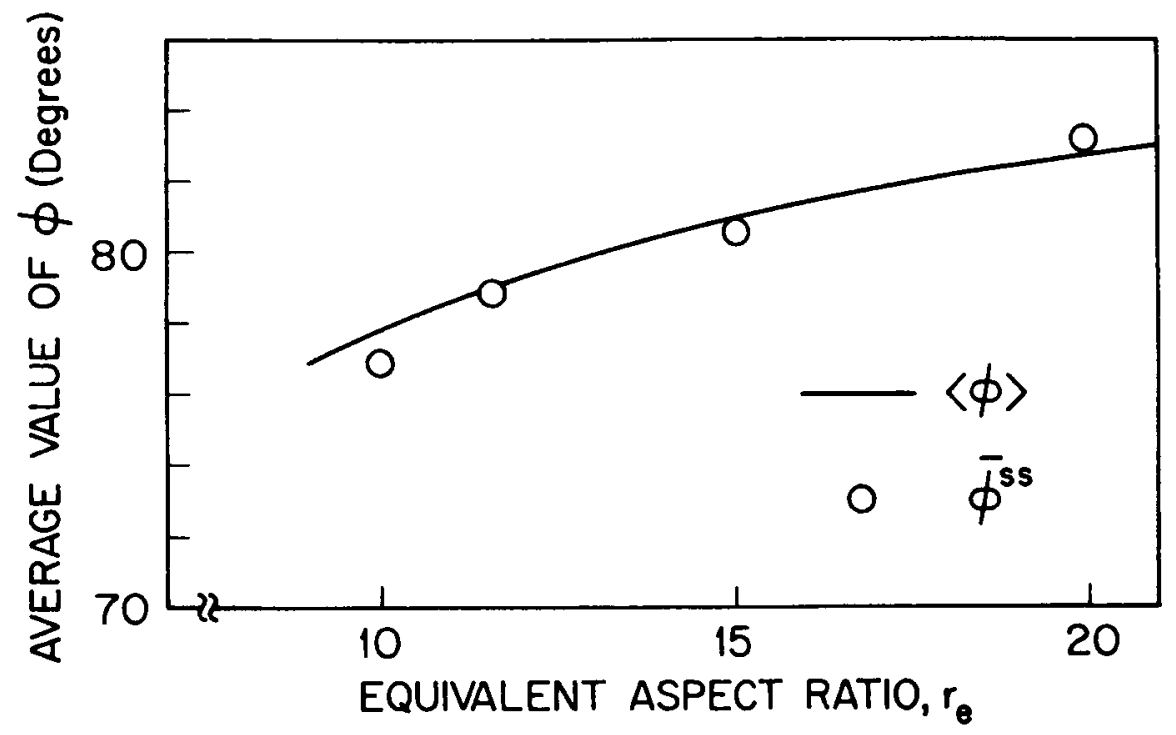

Figure 11. Comparison between the time averaged value of $\phi$ for a single particle $\langle\phi\rangle$ and the number of averaged steady state value of $\phi$ for interacting particles $\phi_{s s^{\circ}}\left(G=0.55^{1}\right)$

Finally, it is noted that the motion of the particles may also be expressed by projections of the particles on the $x_{1} x_{2}, x_{1} x_{3}$ and $x_{2} x_{3}$ planes.

$$
\begin{aligned}
& \overline{X_{1} X_{2}}=\frac{1}{N} \sum_{i=1}^{N} \sin \theta_{i} \\
& \overline{X_{2} X_{3}}=\frac{1}{N} \sum_{i=1}^{N}\left(\cos ^{2} \theta_{i}+\sin ^{2} \theta_{i} \sin ^{2} \theta_{i}\right)^{1 / 2} \\
& \overline{X_{1} X_{3}}=\frac{1}{N} \sum_{i=1}^{N}\left(\cos ^{2} \theta_{i}+\sin ^{2} \theta_{i} \cos ^{2} \theta_{i}\right)^{1 / 2}
\end{aligned}
$$

Typical results illustrating planar projections are presented in Fig. 12.

\section{ACKNOWLEDGEMENTS}

The authors wish to thank Professor W. P. Graebel for his helpful suggestions. This work was supported in part by the Materials Laboratory, Air Force Systems Command, Wright Patterson Air Force Base. 

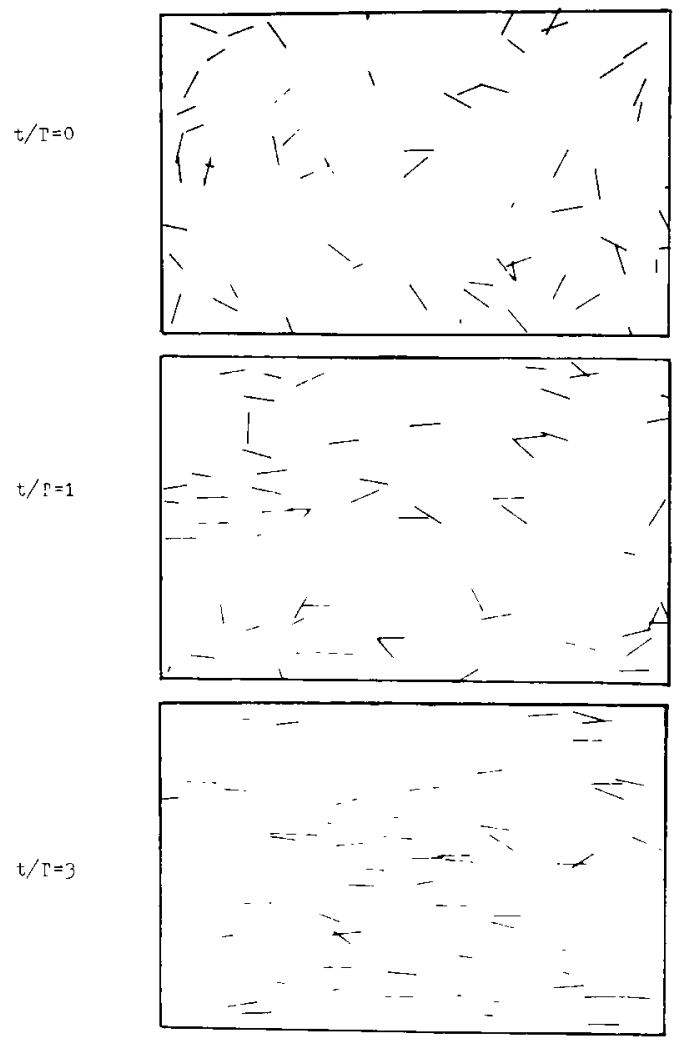

Figure 12. Projections of particles in the $x+x\left\{\right.$ plane. $\left(r_{e}=11.6,0=3.6 \%, n=50 \mathrm{~cm}^{-3}\right.$, $G=0.5 s^{l}, a=0.5$ )

\section{REFERENCES}

1. Goldsmith, H.L. \& Mason, S.G., The microrheology of dispersions, in Rheology, Theory and Applications, (ed. F.R. Eirich), vol. 2., Ch 2, Academic Press, (1967).

2. Cox, R.G. \& Mason, S.G., Suspended particles in fluid flow through tubes, Ann. Rev. Fluid Mech. 3, 291-316, (1971).

3. Leal, L.G., Particle motions in a viscous fluid, Ann. Rev. Fluid Mech. 12, 435-476, (1980).

4. Jeffery, G.B., The motion of ellipsoidal particles immersed in a viscous fluid, Proc. Roy. Soc. $A$ 102, 161-179, (1922).

5. Okagawa, A., Cox, R.G. \& Mason, S.G., The Kinetics of flowing dispersions VI. Transient orientation and rheological phenomena of rods and discs in shear flow, J. Coll. Interface Sci. 45, 303-329, (1973).

6. Dahlquist, G. \& Bjorck, A. Numerical Methods, Prentice Hall, (1974).

7. Trevelyan, B.J. \& Mason, S.G., Particle motions in sheared suspensions, I. Rotations, $J$. Coll. Sci. 6, 354-367, (1951).

8. Okagawa, A. \& Mason, S.G., The Kinetics of flowing dispersions VII. Oscillatory behavior of rods and discs in shear flow. J. Coll. Interface Sci. 45, 330-358, (1973). 
9. Anczurowski, E., Cox, R.G. \& Mason, S.G. The Kinetics of flowing dispersions. IV. Transient orientations of cylinders, J. Coll. Interface Sci. 23, 547-562, (1967).

10. Saffman, P.G., On the motion of small spheroidal particles in a viscous liquid, $J$. Fluid Mech. 1, 540-553, (1956).

11. Taylor, G.I., The motion of ellipsoidal particles in a viscous fluid, Proc. Roy. Soc. A 103, 58-61, (1923).

12. Binder, R.C., The motion of cylindrical particles in viscous flow, J. Appl. Phys. 10, 711-713, (1939).

13. Mason, S.G. Manley, R. St J., Particle motions in sheared suspensions: orientations and interactions of rigid rods, Proc. Roy. Soc. A 238, 117-131, (1956).

14. Anczurowski, E. \& Mason, S.G., Particle motions in sheared suspensions XXIV. Rotation of rigid spheroids and cylinders Trans. Soc. Rheol. 12, 209-215, (1968).

15. Arp, P.A. \& Mason, S.G., Interactions between two rods in shear flow, J. Coll. Interface Sci. $59,378-380,(1977)$.

\section{APPENDIX}

The expressions used in calculating the angular motion of the particles are summarized below.

No collisions. The components of the angular velocity of a single ellipsoidal particle in uniformly sheared flow are [4]

$$
\begin{aligned}
& \omega_{1}=\frac{1}{2}\left(\frac{\partial U_{3}}{\partial \hat{x}_{2}}-\frac{\partial U_{2}}{\partial \hat{x}_{3}}\right) \\
& \omega_{2}=\frac{1}{a^{2}+b^{2}}\left(b^{2} \frac{\partial U_{1}}{\partial \hat{x}_{3}}-a^{2} \frac{\partial U_{3}}{\partial \hat{x}_{1}}\right) \\
& \omega_{3}=-\frac{1}{a^{2}+b^{2}}\left(b^{2} \frac{\partial u_{1}}{\partial \hat{x}_{2}}-a^{2} \frac{\partial u_{2}}{\partial \hat{x}_{1}}\right.
\end{aligned}
$$

The above expressions are for a cartesian coordinate system attached to the particle with the coordinate axes coinciding with the axes of the particle. In order to obtain the angular velocity as a function of position and time, the rotating coordinate system $\left(\hat{\mathbf{x}}_{i}\right)$ must be transformed to a stationary system. In general, the calculations can only be performed by numerical methods.

Closed form solutions can be obtained for particles in plane Couette flow [4]

$$
\begin{aligned}
& \frac{d \phi}{d t}=\dot{\phi}=\frac{G}{r_{e}^{2}+1}\left(r_{e}^{2} \cos ^{2} \phi+\sin ^{2} \phi\right) \\
& \frac{d \theta}{d t}=\dot{\theta}=\frac{G\left(r_{e}^{2}-1\right)}{4\left(r_{e}^{2}+1\right)} \sin 2 \theta \sin 2 \phi
\end{aligned}
$$

Integration of the above two equations yield

$$
\tan \phi=r_{e} \tan \left(\frac{2 \pi t}{T}\right)
$$




$$
\tan \theta=\frac{C r_{e}}{\left(r_{e}^{2} \cos ^{2} \phi+\sin ^{2} \phi\right)^{1 / 2}}
$$

where

$$
T=\frac{2 \pi}{G}\left(r_{e}+\frac{1}{r_{e}}\right)
$$

The value of the integration constant C ("orbit constant") can be determined from the known initial values of $\phi$ and $\theta$. The value of $C$ is assumed to remain the same as the particle moves [15].

Particles Colliding. The angular velocity and the orientations of a particle after a collision are calculated as follows.

(i) Just prior to collision the positions of the centers of masses and the orientations of the major axes of the two colliding particles are known. From this information the position of the contact line $\mathrm{L}$ and the positions of the contact points $C_{A}$ and $C_{B}$ (Fig. 2) are determined.

(ii) The velocity of each contact point before the collision is calculated

$$
V_{c}=V_{t r}+\omega \times \underset{\sim}{\sim}
$$

where $\underline{\mathrm{V}}_{\mathrm{tr}}$ is the translational velocity of the center of mass (and is equal to the instantaneous local fluid velocity), $\omega$, is the angular velocity, and $\mathbf{r}$ is the vector connecting the center of mass and the contact point.

(iii) $\underline{\sim}_{c}$ is decomposed into two components, one being normal and the other one parallel to the contact line L (Fig. 13)

$$
\underline{V}_{c}=\underline{V}_{n}+\underline{V}_{P}
$$

(iv) The normal and parallel velocity components after collision are determined

$$
\begin{aligned}
& \left(\underline{\sim}_{n}^{\prime}\right)_{A}=\left(\underline{\sim}_{n}\right)_{A} \quad\left(\underline{\sim}_{n}^{\prime}\right)_{B}=\left(\underline{\sim}_{n}\right)_{B} \\
& \left(\sim_{P}^{\prime}\right)_{A}=\left(\sim_{P}^{\prime}\right)_{B}={\underset{\sim}{V}}_{P}^{\prime}=\frac{1}{2}\left[\left(\sim_{P}^{V}\right)_{A}+\left(\sim_{P}\right)_{B}\right]
\end{aligned}
$$

The subscripts $\mathrm{A}$ and $\mathrm{B}$ denote the two colliding particles, the prime represents velocities after collision.

(v) The velocities of the contact points after collision are evaluated

$$
\underline{V}_{c}^{\prime}=\underline{V}_{n}^{\prime}+\underline{V}_{P}^{\prime}
$$

(vi) The contribution of the translational velocity is subtracted from $\underline{\sim}_{\text {c }}^{\prime}$ 


\section{Woo Il Lee and George S. Springer}

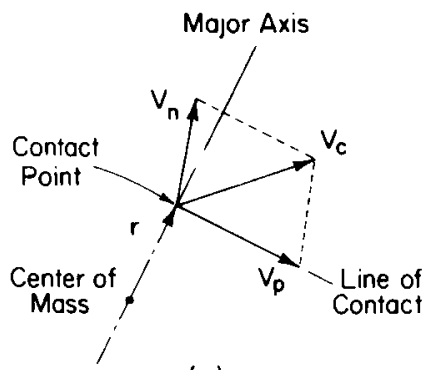

(a)

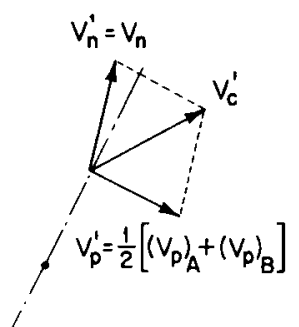

(b)

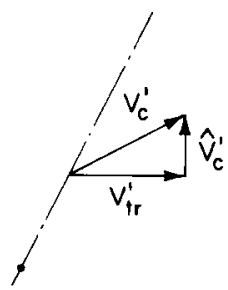

(c)

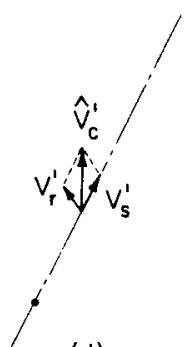

(d)

Figure 13. Vectors representing the motion of the contact point before (Fig. a) and after (Fig. b, c, d) the collision.

$$
\hat{\sim}_{c}^{\prime}=V_{c}^{\prime}-\underline{\sim}_{t r}^{\prime}
$$

and $\hat{\mathbf{V}}^{\prime}{ }_{c}$ is decomposed into two components $\hat{\mathbf{V}}^{\prime}$, and $\hat{\mathbf{V}}^{\prime}{ }_{s}$, where $\hat{\mathbf{V}}^{\prime}$, and $\widetilde{\mathbf{V}}^{\prime}{ }_{s}$ are normal and parallel to the major axis, respectively (Fig. 13).

(vii) The angular velocities of the particles after collision are obtained from the expressions

$$
\begin{aligned}
& \dot{\phi}^{\prime}=\frac{d \phi^{\prime}}{d t}=\frac{1}{r}\left(-V_{r_{1}}^{\prime} \sin \phi+V_{r_{2}}^{\prime} \cos \phi\right) \\
& \dot{\theta}^{\prime}=\frac{d \theta^{\prime}}{d t}=\frac{1}{r}\left(V_{r_{1}}^{\prime} \sin \theta \cos \phi+V_{r_{2}}^{\prime} \sin \theta \sin \phi-V_{r_{3}}^{\prime} \cos \theta\right)
\end{aligned}
$$

$V^{\prime}{ }_{r_{1}}, V^{\prime}{ }_{r_{2}}$, and $V^{\prime}{ }_{r 3}$ are the components of $\hat{\mathbf{V}}^{\prime}{ }_{r}$ in the $x_{1}, x_{2}$ and $x_{3}$ directions. The orientations of the particles after collision are

$$
\phi^{\prime}=\phi+\dot{\phi}^{\prime} \Delta t \text { and } \theta^{\prime}=\theta+\dot{\theta}^{\prime} \Delta t
$$

where $\Delta t$ is the time step used in the calculations. 